\title{
A dual inhibitor overcomes drug-resistant FLT3-ITD acute myeloid leukemia
}

\author{
Peihong Wang ${ }^{\dagger}$, Xinhua Xiao ${ }^{\dagger}$, Yuyin Zhang, Baoyuan Zhang, Donghe Li, Mingzhu Liu, Xi Xie, Chenxuan Liu, \\ Ping Liu* and Ruibao Ren ${ }^{*}$ (D)
}

\begin{abstract}
FLT3 mutations are the most frequently identified genetic alterations in acute myeloid leukemia (AML) and are associated with poor prognosis. Multiple FLT3 inhibitors are in various stages of clinical evaluation. However, resistance to FLT3 inhibitors resulting from acquired point mutations in tyrosine kinase domain (TKD) have limited the sustained efficacy of treatments, and a "gatekeeper" mutation (F691L) is resistant to most available FLT3 inhibitors. Thus, new FLT3 inhibitors against both FLT3 internal tandem duplication (FLT3-ITD) and FLT3-TKD mutations (including F691L) are urgently sought. Herein, we identified KX2-391 as a dual FLT3 and tubulin inhibitor and investigated its efficacy and mechanisms in overcoming drug-resistant FLT3-ITD-TKD mutations in AML. KX2-391 exhibited potent growth inhibitory and apoptosis promoting effects on diverse AML cell lines harboring FLT3-ITD mutations and AC220-resistant mutations at the D835 and F691 residues in TKD and inhibited FLT3 phosphorylation and its downstream signaling targets. Orally administered KX2-391 significantly prolonged the survival of a murine leukemia model induced by FLT3-ITD-F691L. KX2-391 also significantly inhibited the growth of 4 primary AML cells expressing FLT3-ITD and 2 primary AML cells expressing FLT3-ITD-D835Y. Our preclinical data highlight KX2-391 as a promising FLT3 inhibitor for the treatment of AML patients harboring FLT3 mutations, especially refractory/relapsed patients with F691L and other FLT3-TKD mutations.
\end{abstract}

Keywords: Acute myeloid leukemia, FLT3-ITD, FLT3 resistance mutation, KX2-391, AC220

\section{To the editor}

FLT3 mutations occur in more than $30 \%$ of patients with acute myeloid leukemia (AML) and are associated with short relapse-free and overall survival, including internal tandem duplication (ITD) and point mutations within the tyrosine kinase domain (TKD) [1,2]. To date, multiple FLT3 kinase inhibitors have been developed and some are approved for clinical use including sorafenib, Quizartinib (AC220) and Gilteritinib [3]. However,

\footnotetext{
*Correspondence: liupingjize@126.com; ren@brandeis.edu †Peihong Wang and Xinhua Xiao have contributed equally to this work Shanghai Institute of Hematology, State Key Laboratory for Medical Genomics, National Research Center for Translational Medicine (Shanghai), International Center for Aging and Cancer, Collaborative Innovation Center of Hematology, Ruijin Hospital, Shanghai Jiao Tong University School of Medicine, Shanghai, China
}

clinical responses to these drugs are transient because of high rates of relapse and drug resistance after treatment, which contributes to disease progression and poor overall survival $[4,5]$. One particular mechanism for resistance involves acquired additional mutations in the TKD and a "gatekeeper" mutation (F691L) is resistant to most currently available FLT3 inhibitors [6, 7]. Therefore, finding effective compounds to overcome the drug resistance caused by F691L and other mutations is an urgent problem. Herein, we identified KX2-391 as a FLT3 inhibitor and evaluated its activity against FLT3-ITD-TKD mutations using in vitro and in vivo models.

We used molecular docking simulations to computationally screen 1487 small molecule ligands from the L3400 Clinical Compound Library and identified KX2391 as a candidate FLT3 inhibitor (Additional file 1: original author(s) and the source, provide a link to the Creative Commons licence, and indicate if changes were made. The images or other third party material in this article are included in the article's Creative Commons licence, unless indicated otherwise in a credit line to the material. If material is not included in the article's Creative Commons licence and your intended use is not permitted by statutory regulation or exceeds the permitted use, you will need to obtain permission directly from the copyright holder. To view a copy of this licence, visit http://creativecommons.org/licenses/by/4.0/. The Creative Commons Public Domain Dedication waiver (http://creativeco mmons.org/publicdomain/zero/1.0/) applies to the data made available in this article, unless otherwise stated in a credit line to the data. 


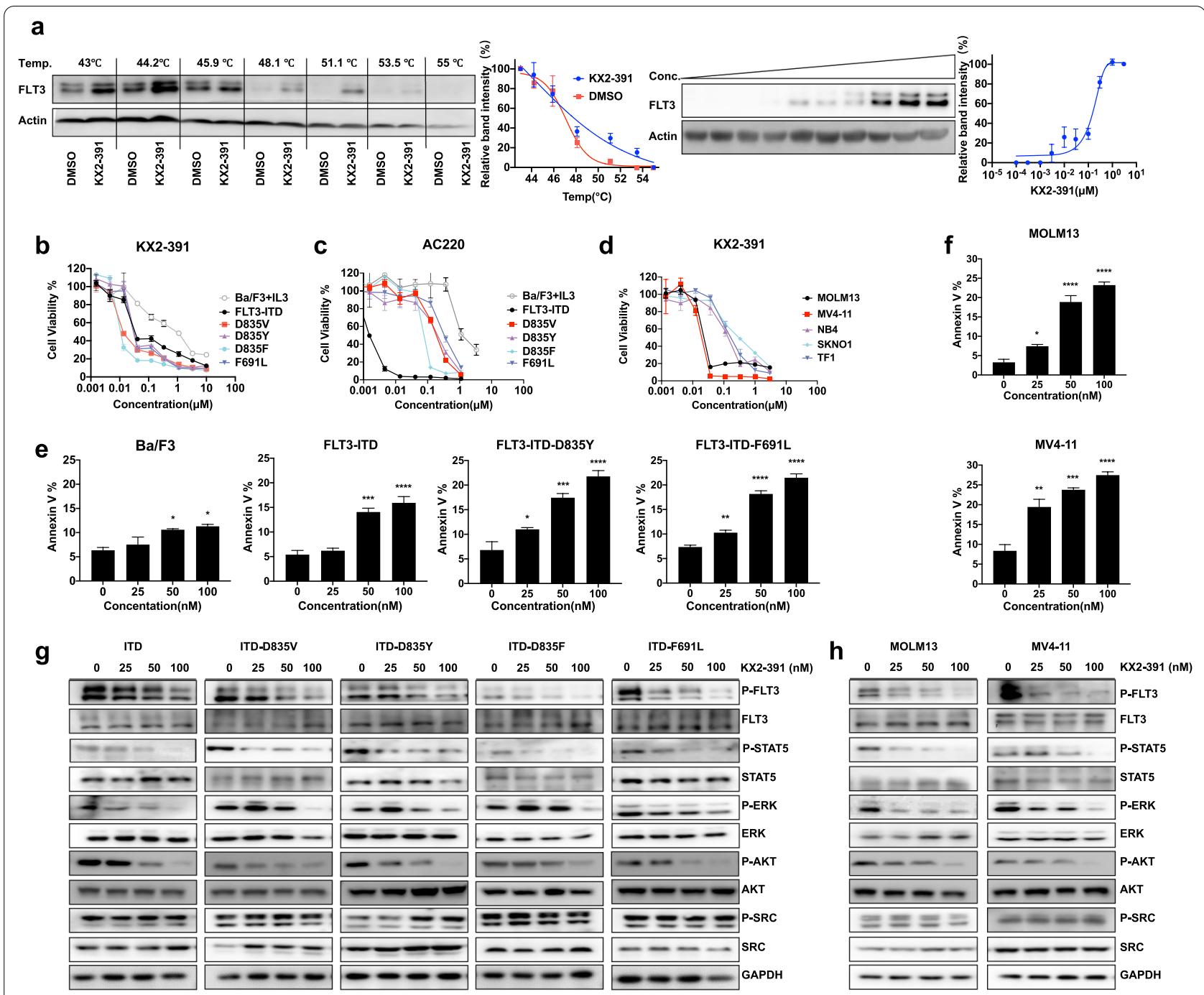

Fig. 1 KX2-391 is active against ITD-TKD resistance-causing FLT3 mutations and blocks FLT3 signaling in FLT3-ITD and FLT3-ITD-TKD cells. a Quantification of cellular thermal shift assay was made using western blot Ba/F3 cells expressing FLT3-ITD cells were treated with KX2-391 (1 $\mu$ M) for $1 \mathrm{~h}$, and temperatures between 43 and $55^{\circ} \mathrm{C}$ were defined to perform the test. $\mathrm{KX} 2-391$ were treated based on 10 different concentrations for $1 \mathrm{~h}$ at $51^{\circ} \mathrm{C}$. Data were normalized by setting the highest and lowest value in each set to $100 \%$ and $0 \%$, respectively. Data were obtained in the presence of the KX2-391 (blue circle) as the positive control and DMSO (red square) as the negative control. b Normalized cell viability of Ba/F3 cells expressing FLT3-ITD TKD mutations after a $48 \mathrm{~h}$ exposure to various concentrations of KX2-391 and AC220 (c), measured using CellTiter Glo assays (error bars represent the SD of 3 or more independent experiments). $\mathbf{d}$ Viability of FLT3-ITD mutated cells (MV4-11, MOLM-13), and FLT3 nonmutated cells (NB4, SKNO1, TF1) treated with various concentrations of KX2-391 for 48 h, measured by CellTiter Glo assays. e Ba/F3 cells expressing FLT3-ITD TKD mutations were treated with different concentrations of KX2-391 for $24 \mathrm{~h}$ and then parental Ba/F + IL3, FLT3-ITD, FLT3-ITD-D835Y, and FLT3-ITD-F691L cells, as well as $\mathbf{f M O L M 1 3}$ and MV4-11 cells were examined by flow cytometry (Annexin V). $\mathbf{g}$ Ba/F3 cells expressing FLT3-ITD, FLT3-ITD-D835Y, FLT3-ITD/F691L, FLT3-D835V, and FLT3-D835F, as well as FLT3-ITD positive human leukemia cell lines h MOLM13 and MV4-11 were incubated for $12 \mathrm{~h}$ with the indicated concentrations of KX2-391 (based on the IC50 values) and subsequently examined by western blotting using antibodies against FLT3/P-FLT3, STAT5/P-STAT5, ERK/P-ERK, AKT/P-AKT and SRC/P-SRC. GAPDH was used as a loading control. All values represent the mean $\pm S D$ of three independent experiments. ${ }^{*} P<0.05$; ${ }^{*} P<0.01 ;{ }^{* *} P<0.001 ;{ }^{* * *} P<0.0001$ versus the control

Table S1). Previous studies reported KX2-391 was an SRC/tubulin dual inhibitor and exhibited anticancer activities in some tumors [8]. Our modeling predicted the interaction of KX2-391 with FLT3's L616, V624 and E661 residues (Additional file 2: Fig. S1). We further confirmed the interactions between FLT3 and KX2-391 by cellular thermal shift assay. Compared with DMSO, an obvious thermal shift of the melting curve was detected in the KX2-391-treated sample. The thermal stability of FLT3 protein was increased by KX2-391 in a dose-dependent manner (Fig. 1a). We evaluated the inhibitory activity of KX2-391 against different forms 


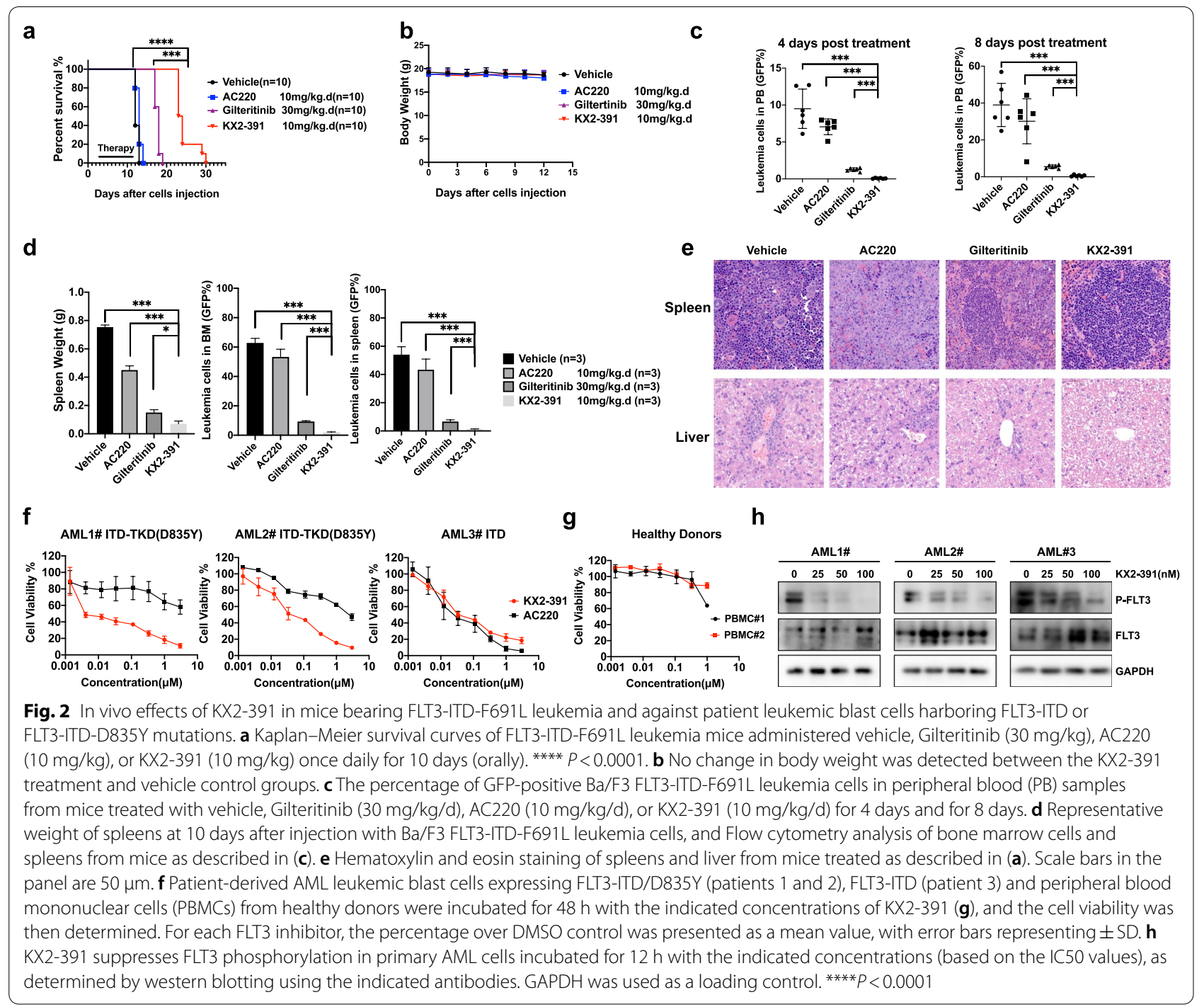

of FLT3 in Ba/F3 cells and found it potently inhibited the growth of FLT3-ITD-expressing Ba/F3 cells and all tested cells expressing FLT3-ITD-TKD mutations previously linked with drug resistance to FLT3 inhibitors, such as AC220 (Fig. 1b, c, Additional file 1: Table S2). Notably, Ba/F3-ITD-F691L cells were tenfold more sensitive to KX2-391 than parental $\mathrm{Ba} / \mathrm{F} 3$ cells $(0.032 \mu \mathrm{M}$ vs $0.372 \mu \mathrm{M})$. KX2-391 also displayed higher inhibitory efficacy on human leukemia cell lines harboring FLT3-ITD (MV4-11 and MOLM13) than that on FLT3 nonmutated leukemia cell lines (Fig. 1d). We observed dose-dependent induction of apoptosis in $\mathrm{Ba} / \mathrm{F} 3$ cells expressing FLT3-ITD, FLT3-ITD-D835Y and FLT3-ITD-F691L as well as in two FLT3-ITD positive AML cell lines (Fig. 1e, f). KX2-391 prominently inhibited the phosphorylation of FLT3 and downstream targets STAT5, ERK and AKT in FLT3-ITD,
FLT3-ITD-F691L-expresssing Ba/F3 cells and other cells of our assay panel (Fig. 1g, h). Recalling that KX2391 has to date understood as an SRC/tubulin inhibitor [9], we monitored SRC phosphorylation and assessed KX2-391's effects on microtubule morphology. KX2391 treatment did not alter phosphorylation of SRC in FLT3 mutant cells (Fig. 1g, h). We did detect disrupted tubulin polymerization in MOLM13 cells treated with KX2-391 and with various known tubulin inhibitors (Additional file 2: Fig. S2). Excluding a direct impact of tubulin modulation, assays showed that the known tubulin inhibitor Vincristine did not affect phosphorylation of FLT3 or its downstream targets (Additional file 2: Fig. S3).

Next, we used the previously described Ba/F3-ITDF691L leukemia model to measure the efficacy of KX2391 in vivo [10]. KX2-391 showed a significant benefit 
on median survival, prolonging the survival period from 12 days in the control group to 23.5 days (Fig. 2a, $\mathrm{P}<0.0001$ ). No significant weight loss (Fig. $2 \mathrm{~b}$ ) or other signs of toxicity were observed during treatment. Compared to the vehicle control, AC220 and Gilteritinib groups, KX2-391-treated mice had fewer leukemia cells (in peripheral blood, spleen, and bone marrow) (Fig. 2c, d) and had smaller spleens (Fig. 2d). H\&E staining demonstrated that KX2-391 treatment significantly reduced AML cell infiltration into the spleen and liver (Fig. 2e).

We also evaluated the anti-leukemia effects of KX2-391 on primary AML cells isolated from 6 newly diagnosed FLT3-ITD AML patients (Additional file 1: Table S3). KX2-391 effectively reduced cell viability in 4 primary blasts with FLT3-ITD mutations (comparable with AC220's effect). It also significantly inhibited the growth of 2 primary AML cells expressing FLT3-ITD-D835Yboth of which were resistant to AC220 (Fig. 2f, Additional file 2: Fig. S4). KX2-391 did not affect the growth of healthy peripheral blood mononuclear cells at the same concentrations (Fig. 2g). We confirmed that KX2391 inhibited FLT3 phosphorylation in primary AML cells (Fig. 2h).

Collectively, we show that KX2-391 is an FLT3/tubulin inhibitor that exerts strong therapeutic effects both in vitro and in vivo against FLT3-ITD and drug resistant TKD mutations including FLT3-ITD-F691L, which is understood as the most difficult mutation to overcome clinically. Considering the detected therapeutic effects and its apparently minimal toxicity [11, 12], KX2-391 may become a useful second-line drug suitable for treating some of the most clinically challenging AML cases.

\begin{abstract}
Abbreviations
AML: Acute myelogenous leukemia; BMCs: Bone marrow cells; FLT3: FMS-like tyrosine kinase 3; ITD: Internal tandem duplication; TKD: Tyrosine kinase domain; ERK: Extracellular signal regulated kinase; STAT: Signal transducer and activator of transcription; VCR: Vincristine; IC50: Half maximal inhibitory concentration; GFP: Green fluorescent protein; APC: Allophycocyanin; H\&E: Hematoxylin and eosin; SDS: Sodium dodecyl sulfate.
\end{abstract}

\section{Supplementary Information}

The online version contains supplementary material available at https://doi. org/10.1186/s13045-021-01098-y.

Additional file 1: Table S1. 14 small molecule inhibitors are in clinical trials of leukemia. Table S2. Inhibitory activity of KX2-391 against FLT3 mutant and FLT3 nonmutated cell lines. Table S3. Patient information (DOCX $18 \mathrm{~kb}$ )

Additional file 2: Figure S1. Computational modeling of KX2-391 binding with wild-type FLT3. (a) An overview of the docking results of KX2-391 with FLT3 (Protein Data Bank: 5X02); two orthogonal views are shown. (b) Close-up of the KX2-391-FLT3 model, highlighting the hydrogen bond formed by residues LEU-616 and GLU-661, the predicted KX2-391 binding site are not included Phe691. Figure S2. KX2-391 disrupted microtubules in MOLM13 cells. MOLM13 cells were treated with dimethyl sulfoxide
(DMSO, control), 100 nM Paclitaxel, 100 nM Vincristine, 100 nM Colchicine,

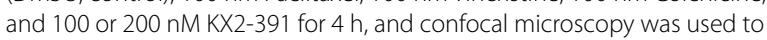
observe the signal corresponding to a-tubulin (green); DNA was counterstained with DAPI (blue). Figure S3. Vincristine (VCR) does not inhibit FLT3 signaling in FLT3-ITD or FLT3-ITD-TKD cells. Ba/F3 cells expressing FLT3-ITD, FLT3-ITD-D835Y, or FLT3-ITD-F691L, and human cell lines MOLM13 and MV4-1 1 were incubated for $12 \mathrm{~h}$ with the indicated concentrations of VCR (based on the IC50 values) and subsequently examined by western blotting using the indicated antibodies. GAPDH was used as a loading control. Figure S4. Potent inhibition of KX2-391 against patient leukemic blast cells harboring FLT3-ITD. Patient-derived AML leukemic blast cells expressing FLT3-ITD (patients 4-6) were incubated for $48 \mathrm{~h}$ with the indicated concentrations of KX2-391 and AC220, and the cell viability of blast cells was then determined with CellTiter Glo assays. For each FLT3 inhibitor, the percentage over DMSO control was presented as a mean value, with error bars representing $\pm S D$.

Additional file 3. Detailed materials and methods.

\section{Acknowledgements}

The authors thank Jin Yi for her assistance with cell sorting. This work was supported by the Natural Science Foundation of China (81870112 to R.R. and 81970134 to P. L.), the Key Project of Natural Science Foundation of China (81530006 to R.R.), the Samuel Waxman Cancer Research Foundation (to R.R.), Shanghai Science and Technology Development Funds (No. $20 Z 11900200$ to R.R. $18 Z R 1423600$ to P.L.), Shanghai Collaborative Innovation Program on Regenerative Medicine and Stem Cell Research (2019CXJQ01 to R.R.) and the Innovative Research Team of High-level Local Universities in Shanghai (to R.R.).

\section{Authors' contributions}

P.H.W, X.H.X, Y.Y.Z, P.L and R.B.R designed the experiment, analyzed data and wrote the paper. P.H.W, X.H.X, Y.Y.Z performed the experiments and analyzed data. B.Y.Z helped to analyzed data. D.H.L, M.Z.L, X.X and C.X.L helped to perform the animal experiments and collect patient samples. R.B.R and P.L supervised the study. All authors read and approved the final manuscript.

\section{Availability of data and materials}

All data generated or analyzed during this study are included in this published article and its supplementary information files.

\section{Declarations}

\section{Ethical approval and consent to participate}

All animal experiments were approved by The Animal Care \& Welfare Committee of Shanghai Jiao tong University School of Medicine. Patient samples preparation was approved by the Institutional Review Board of the Ruijin Hospital affiliated to Shanghai Jiao tong University School of Medicine.

\section{Consent for publication}

Not applicable.

\section{Competing interests}

The authors declare no competing interests.

Received: 11 April 2021 Accepted: 26 May 2021

Published online: 03 July 2021

\section{References}

1. Lemonnier F, Inoue S, Mak TW. Genomic classification in acute myeloid leukemia. N Engl J Med. 2016;375(9):900-900.

2. Ley TJ, Miller C, Ding L, Raphael BJ, Mungall AJ, Robertson AG, Hoadley K, Triche TJ Jr, Laird PW, Baty JD, et al. Genomic and epigenomic landscapes of adult de novo acute myeloid leukemia. N Engl J Med. 2013;368(22):2059-74.

3. Wu M, Li C, Zhu X. FLT3 inhibitors in acute myeloid leukemia. J Hematol Oncol. 2018;11(1):133. 
4. Smith CC, Wang Q, Chin C-S, Salerno S, Damon LE, Levis MJ, Perl AE, Travers KJ, Wang S, Hunt JP, et al. Validation of ITD mutations in FLT3 as a therapeutic target in human acute myeloid leukaemia. Nature. 2012;485(7397):260-U153

5. Smith CC, Paguirigan A, Jeschke GR, Lin KC, Massi E, Tarver T, Chin C-S, Asthana S, Olshen A, Travers KJ, et al. Heterogeneous resistance to quizartinib in acute myeloid leukemia revealed by single-cell analysis. Blood. 2017;130(1):48-58.

6. Eguchi M, Minami Y, Kuzume A, Chi S. Mechanisms underlying resistance to FLT3 inhibitors in acute myeloid leukemia. Biomedicines. 2020;8(8):245.

7. Smith CC, Zhang C, Lin KC, Lasater EA, Zhang Y, Massi E, Damon LE, Pendleton M, Bashir A, Sebra R, et al. Characterizing and overriding the structural mechanism of the quizartinib-resistant FLT3 "gatekeeper" F691L mutation with PLX3397. Cancer Discov. 2015;5(6):668-79.

8. Niu L, Yang J, Yan W, Yu Y, Zheng Y, Ye H, Chen Q, Chen L. Reversible binding of the anticancer drug $\mathrm{KXO}$ (tirbanibulin) to the colchicinebinding site of $\beta$-tubulin explains KXO1's low clinical toxicity. J Biol Chem. 2019;294(48):18099-108.

9. Anbalagan M, Carrier L, Glodowski S, Hangauer D, Shan B, Rowan BG. KX-01, a novel Src kinase inhibitor directed toward the peptide substrate site, synergizes with tamoxifen in estrogen receptor alpha positive breast cancer. Breast Cancer Res Treat. 2012;132(2):391-409.
10. Ju HQ, Zhan G, Huang A, Sun Y, Wen S, Yang J, Lu WH, Xu RH, Li J, Li Y, et al. ITD mutation in FLT3 tyrosine kinase promotes Warburg effect and renders therapeutic sensitivity to glycolytic inhibition. Leukemia. 2017;31(10):2143-50.

11. Kasner MT, Ritchie EK, Cutler D, Fetterly GJ, Kramer D, Hangauer D, Thompson JE. A phase $1 \mathrm{~b}$ dose escalation study to evaluate safety, tolerability and pharmacokinetics of oral monotherapy with KX2-391 in elderly subjects with acute myeloid leukemia who are refractory to or have declined standard induction therapy. J Clin Oncol. 2017;35(15_suppl):7043.

12. Antonarakis ES, Heath El, Posadas EM, Yu EY, Harrison MR, Bruce JY, Cho SY, Wilding GE, Fetterly GJ, Hangauer DG, et al. A phase 2 study of KX2-391, an oral inhibitor of Src kinase and tubulin polymerization, in men with bone-metastatic castration-resistant prostate cancer. Cancer Chemother Pharmacol. 2013;71(4):883-92.

\section{Publisher's Note}

Springer Nature remains neutral with regard to jurisdictional claims in published maps and institutional affiliations.
Ready to submit your research? Choose BMC and benefit from:

- fast, convenient online submission

- thorough peer review by experienced researchers in your field

- rapid publication on acceptance

- support for research data, including large and complex data types

- gold Open Access which fosters wider collaboration and increased citations

- maximum visibility for your research: over $100 \mathrm{M}$ website views per year

At BMC, research is always in progress.

Learn more biomedcentral.com/submissions 